\title{
Higher-order corrections in non-minimal supersymmetric models
}

\section{P. Athron}

School of Physics and Astronomy, Monash University, Victoria 3800, Australia

\section{P. Diessner}

Institut für Kern- und Teilchenphysik, TU Dresden, Germany

\section{J. Kalinowski}

Faculty of Physics, University of Warsaw, Poland

\section{W. Kotlarski}

Institut für Kern- und Teilchenphysik, TU Dresden, Germany

\section{J. Park}

Quantum Universe Center, KIAS, Seoul, Republic of Korea

\section{T. Steudtner}

Institut für Kern- und Teilchenphysik, TU Dresden, Germany

\section{Stöckinger*}

Institut für Kern- und Teilchenphysik, TU Dresden, Germany

\section{A. Voigt}

DESY theory group, DESY, Hamburg, Germany

The first topic of these proceedings is the phenomenology of the Minimal R-symmetric supersymmetric standard model (MRSSM) and the question whether this model can accommodate the mass of the Higgs boson observed at the LHC. It is shown that the answer is affirmative, and the MRSSM can simultaneously accommodate the Higgs boson mass, electroweak precision observables, LHC data, and dark matter. Suitable mass spectra are promising in view of future LHC searches. The second topic is discussion of an automatic effective field theory-calculation of the Higgs boson mass implemented in FlexibleSUSY. It is shown that a specific choice of the matching conditions significantly improves the calculation and combines virtues of effective field theory and fixed-order calculations. Theory uncertainties of different calculations are assessed.

Loops and Legs in Quantum Field Theory - LL 2016,

Leipzig, Germany

\footnotetext{
*Speaker.
} 


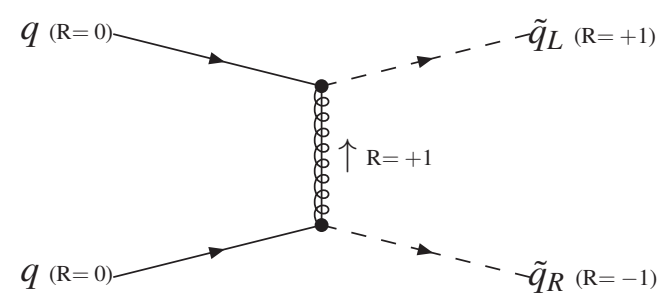

Figure 1: Feynman diagram for like-sign squark pair production in the MRSSM. The solid/curly line denotes a Dirac gluino. The $\mathrm{R}$ charges of the left- and right-handed squark and the gluino are as indicated.

\section{Introduction}

In these proceedings we discuss two topics with one common theme: precision Higgs-boson mass predictions in supersymmetric models. The first topic is the phenomenology of the Minimal R-symmetric supersymmetric standard model (MRSSM) and the question whether this model can accommodate the mass of the Higgs boson observed at the LHC [1, 2, 3]. The second topic is discussion of an automatic effective field theory-calculation of the Higgs boson mass implemented in FlexibleSUSY [4].

\section{The Minimal R-symmetric supersymmetric standard model and the Standard Model-like Higgs boson}

The Minimal R-symmetric supersymmetric standard model (MRSSM) [5] is a very appealing, alternative supersymmetric model. It is distinct from the usual minimal supersymmetric standard model (MSSM), and it does not possess an MSSM-limit.

Figure 1 shows a tree-level Feynman diagram for like-sign squark production. It illustrates many essential features of the MRSSM. The MRSSM is based on an additional continuous Rsymmetry, which is required to be an exact symmetry under which all standard model (SM) particles are assumed to be uncharged. The supersymmetry (SUSY) algebra then fixes uniquely the R-charges of all SUSY particles. Scalar members of chiral (anti-chiral) SUSY multiplets like left(right-)handed squarks have $\mathrm{R}=+1(\mathrm{R}=-1)$, the gluino (like all other gauginos) is a Dirac fermion with $\mathrm{R}=+1$. The process shown in Fig. 1 is therefore allowed by R-symmetry, but other processes such as $q q \rightarrow \tilde{q}_{L} \tilde{q}_{L}$ or $q q \rightarrow \tilde{q}_{R} \tilde{q}_{R}$ are forbidden in the MRSSM (but allowed in the MSSM). As a result, the total cross section for SUSY particle production in the MRSSM is smaller than in the MSSM, and lighter SUSY masses can still be compatible with LHC data.

Since gauginos and Higgsinos have R-charge, they are Dirac fermions. So they involve additional fermionic degrees of freedom, which have new scalar superpartners. Hence the MRSSM contains scalars in the adjoint representation of $\mathrm{SU}(3)_{C} \times \mathrm{SU}(2)_{W} \times \mathrm{U}(1)_{Y}$ ("sgluons", "Higgs triplet", and "Higgs singlet"), and two additional Higgs doublets ("R-Higgs doublets").

In the MRSSM it is not immediately obvious whether the observed, high mass of the SMlike Higgs boson can be accommodated. At tree-level, the MSSM-like Higgs states can mix with the additional, neutral components of the Higgs triplet and singlet. Hence the tree-level Higgs boson mass is reduced compared to the MSSM. Conversely, the phenomenological requirement of a high Higgs boson mass motivates small mixing with the Higgs triplet and singlet. Looking at 
the appropriate mass matrix shows that this translates into the requirement that the Higgsino and electroweak gaugino masses should be rather small, i.e. of the order of few $100 \mathrm{GeV}$.

At the one-loop level there is a further difficulty compared to the MSSM. In the MSSM, the Higgs boson mass is strongly pushed upwards by top/stop loops, particularly in case of large stop left-right mixing. However, stop left-right mixing is forbidden in the MRSSM since it violates R-charge conservation (see the squark R-charges in Fig. 1).

However, there are also positive contributions of the MRSSM to the SM-like Higgs boson mass. The superpotential contains trilinear terms $\hat{H}_{u} \hat{S} \hat{R}_{u}$ and $\hat{H}_{u} \hat{T} \hat{R}_{u}$ (where the hat denotes superfields; $S, T$ and $R_{u}$ denote the Higgs singlet, triplet and up-R-Higgs), and similar terms involving $\hat{H}_{d}$. These superpotential terms have the same structure as the top Yukawa term, and they lead to one-loop contributions to the Higgs boson mass, which are approximately

$$
\left(\Delta M_{h}\right)^{2} \approx \frac{2 v^{2}}{16 \pi^{2}}\left(\frac{\Lambda^{2} \lambda^{2}}{2}+\frac{4 \lambda^{4}+4 \lambda^{2} \Lambda^{2}+5 \Lambda^{4}}{4} \log \frac{m_{\text {soft }}^{2}}{m_{D}^{2}}\right)
$$

Here the couplings of these superpotential terms are called $\lambda$ and $\Lambda$, and the scalar masses and Dirac fermion masses are set to $m_{\text {soft }}$ and $m_{D}$, respectively. If $\lambda$ and/or $\Lambda$ are assumed to be of the same order as the top Yukawa coupling, then these loop contributions can easily push up the Higgs boson mass to values as high as in the MSSM.

At the two-loop level there are additional positive corrections, most notably from the MRSSMspecific sgluons, the new scalar superpartners of the Dirac gluino. The sgluons couple with the strong gauge coupling, and mixed stop/sgluon-two-loop diagrams can further push up the Higgs boson mass by more than $5 \mathrm{GeV}$.

Thanks to these positive MRSSM loop corrections, there are viable parameter regions of the MRSSM in which the Higgs boson mass observed at the LHC is accommodated. Simultaneously, it is possible to achieve agreement with electroweak precision observables, particularly the mass of the $\mathrm{W}$ boson. Figure 2 shows a corresponding sample parameter point which is in agreement with the measured Higgs boson mass at the LHC and with further constraints from LHC searches and electroweak precision measurements. In Ref. [3] it was shown that it is also possible to achieve this agreement with experimental data even in the presence of a very light singlet-like Higgs boson and a very light Dirac singlino/bino state. In this case even the observed relic dark matter density can be explained, and future LHC measurements have the potential to discover additional, very light states.

\section{EFT- versus fixed order calculations of Higgs masses with FlexibleSUSY}

The experimental precision of the LHC Higgs boson mass measurement has already reached the per-mille level. In contrast, despite more than two decades of intense effort, the theory uncertainty of the most advanced predictions of Higgs boson masses in SUSY models is at least ten times higher. Hence it is very important to increase the level of theory precision, but also to check and if possible improve the reliability of theory error estimates. Furthermore, a high theory precision is not only important in the MSSM but also in competing alternative models such as the MRSSM and others. 


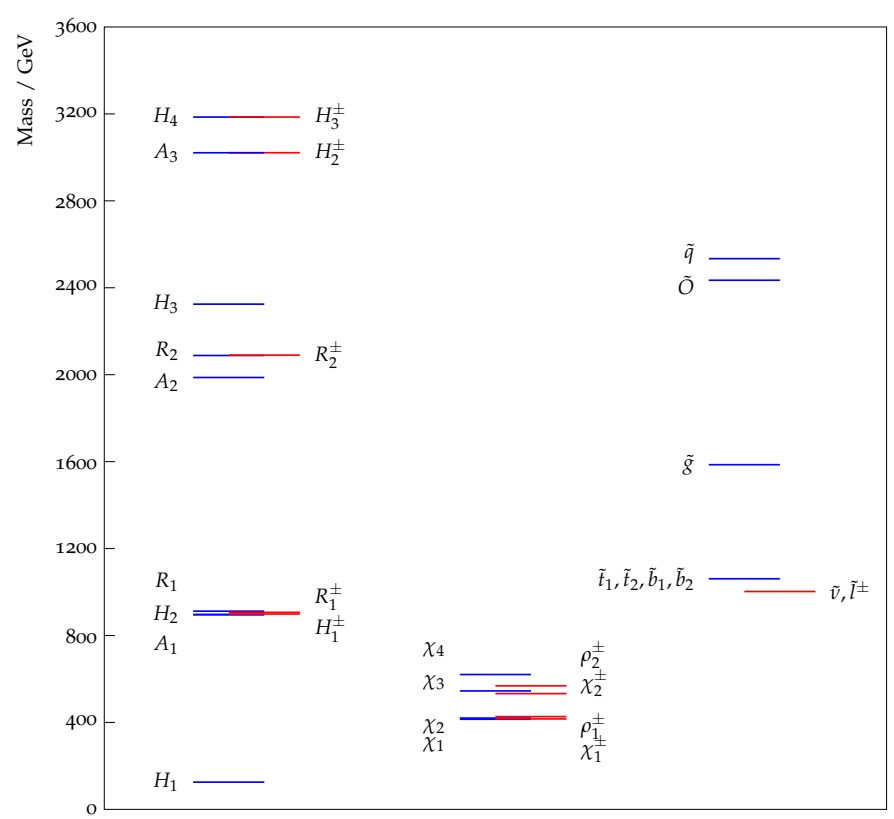

Figure 2: Mass spectrum of a sample parameter point, BMP1 defined in Ref. [1], which is in agreement with the measured Higgs boson mass at the LHC and with further constraints from LHC searches and electroweak precision measurements.

In general, two main approaches to compute the Higgs boson mass can be distinguished. The most obvious way is a fixed-order computation of the Higgs self energy (this can be done in the $\overline{\mathrm{DR}}$-renormalization scheme, the on-shell scheme or a mixed scheme). A second way is based on effective field theory (EFT) techniques. The SUSY particles are assumed to be heavy, and they are integrated out at the heavy scale. Below that scale, the parameters in the low-energy EFT (here we take this to be the Standard Model) are run down to the electroweak scale, at which the Higgs boson mass is computed. In this EFT-approach, large logarithms of the heavy SUSY mass scale are resummed.

These considerations motivate to implement several different Higgs mass calculation algorithms into the framework FlexibleSUSY [6]. FlexibleSUSY is a framework which allows to generate c++ programs which compute mass spectra and further observables in minimal and nonminimal SUSY models. The models are specified by SARAH model files, and analytical expressions for $\beta$ functions and self energies are obtained from SARAH [7]. Within FlexibleSUSY, many calculations could be implemented, the most important of which are:

- In the MSSM, there are several automatically generated Higgs mass computations, and several MSSM-specific modified computations: (1) the original out-of-the box computation (fixed-order two-loop calculation in the $\overline{\mathrm{DR}}$-scheme (and in the gaugeless limit), essentially equivalent to the one in Softsusy [8]); (2) a variant which is essentially equivalent to the one in Spheno [9]; (3) an automatically generated effective-field theory (EFT) computation (with one-loop matching and three-loop running); and (4) a variant which is essentially equivalent to the one in SUSYHD [10] (two-loop matching and three-loop running). 


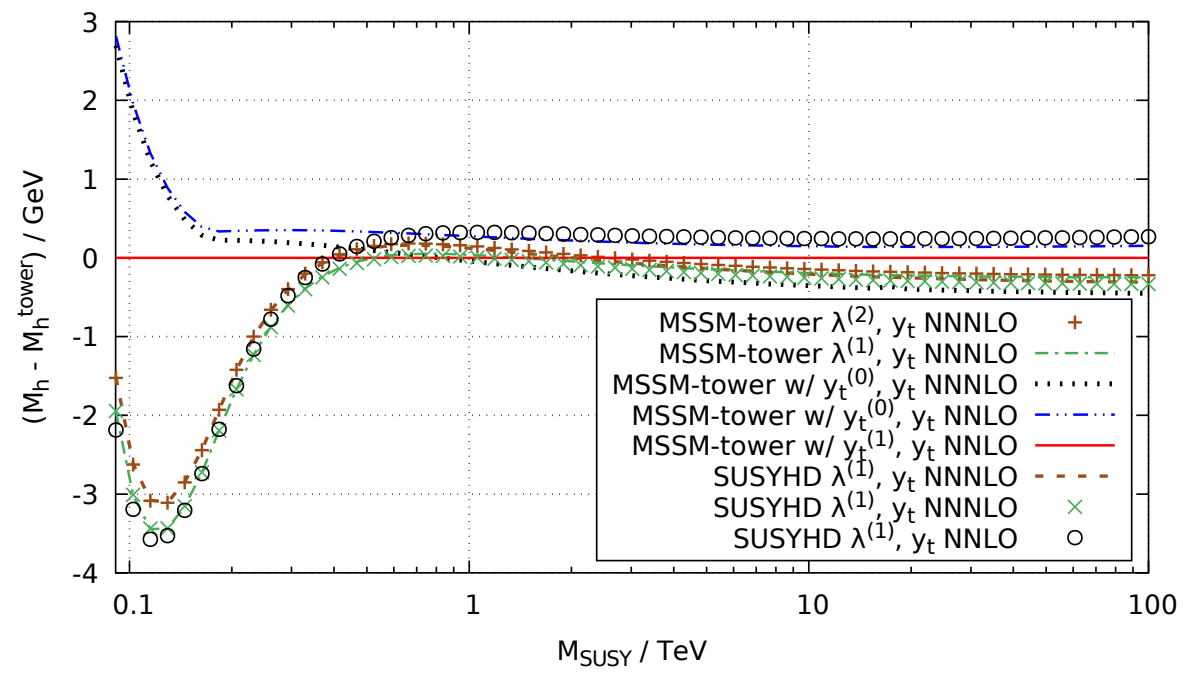

Figure 3: SM-like Higgs boson mass in the MSSM for a common SUSY mass scale and vanishing sfermion mixing in the FlexibleSUSY version MSSM-Tower, in SUSYHD with several options, a versions of MSSMTower which mimicks SUSYHD, and in several variants of the tower in which SUSYHD-like code is replaced step by step by the original tower-code, see text.

- In non-minimal models, there are two automatically generated calculations: (1) the original fixed-order one-loop calculation; (2) an EFT computation with one-loop matching and threeloop running.

With these computations at hand, many verifications of the code are possible. But more importantly it becomes possible to study in detail the influences of various terms and design choices in the computations. Such studies and comparisons are greatly aided and systematized by the fact that all calculations are based on one code, which is under full control.

Now we briefly discuss the conclusions that can be drawn from such comparisons for the case of the MSSM. Figure 3 analyzes the differences of the predictions between the Higgs mass prediction of our automatically generated EFT-calculation in FlexibleSUSY ("MSSM-tower", red solid line) and of the public program SUSYHD with its maximum precision (dashed brown line). The SUSY mass parameters are all set to the common scale $M_{\text {SUSY }}$, and sfermion mixing is set to zero.

We have implemented a variant of MSSM-tower in FlexibleSUSY, which essentially replicates SUSYHD ("MSSM-tower $\lambda^{(2)}, y_{t}$ NNNLO, brown pluses). Then we have implemented versions of MSSM-tower, in which SUSYHD-like code is replaced step by step by the original MSSMtower code. In this way, we see that the essential difference between MSSM-tower and SUSYHD is caused by four design choices: (1; green dot-dashed line) different loop order of the matching at the SUSY scale: this difference is negligible due to the well-known fact that the two-loop matching corrections are negligible in the considered parameter scenario, (2; black dottet line) different type of matching condition at the SUSY scale, (3; blue dot-dashed line) different order of weak-scale matching the top-quark mass to experiment: this shifts the Higgs mass by about $0.5 \mathrm{GeV}$, as has already been stressed in [10], (4; red line) inclusion of higher orders in the top-quark Yukawa 


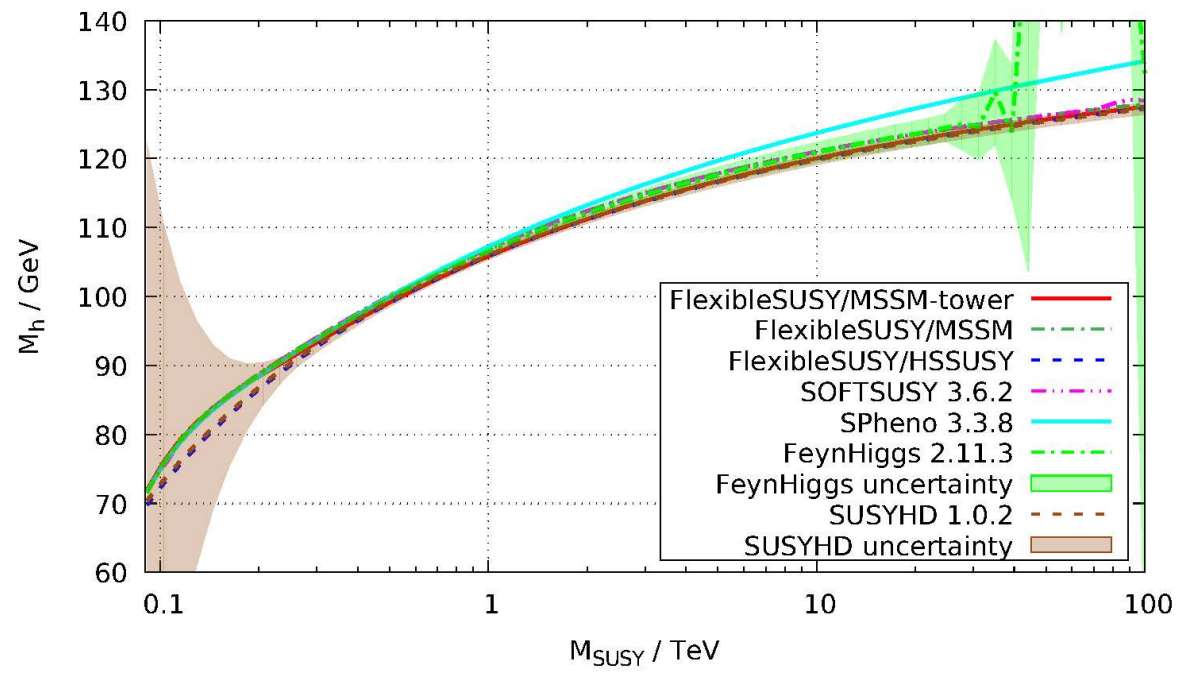

Figure 4: SM-like Higgs boson mass in the MSSM for a common SUSY mass scale and vanishing sfermion mixing in various public codes and three versions of FlexibleSUSY: the original one, a version which mimicks SUSYHD ("HSSUSY"), and MSSM-Tower.

coupling at the SUSY scale.

The crucial difference to be discussed here is (2), corresponding to the choice of the type of matching condition at the SUSY scale. In SUSYHD, a "pure EFT" choice is made, and particularly the quartic Higgs self coupling $\lambda$ in the Standard Model is obtained from matching the Higgs fourpoint function at zero momentum. In this way, already at the one-loop level, power-suppressed terms of order $M_{\text {weak }}^{2} / M_{\text {SUSY }}^{2}$ are neglected. In contrast, MSSM-tower determines $\lambda$ by matching the Higgs boson pole mass between the full SUSY theory and the EFT. In this way, the final result for the Higgs boson mass in MSSM-tower is completely correct at the one-loop level, and powersuppressed terms of order $M_{\text {weak }}^{2} / M_{\text {SUSY }}^{2}$ are neglected only at the two-loop level.

As the difference between the black dotted and green dot-dashed lines shows, the numerical impact of the change of $\lambda$-matching condition is small at high $M_{\mathrm{SUSY}}$, but quite large at small $M_{\text {SUSY }}$. In any case, by using pole-mass matching one part of the theory error is significantly reduced.

Figure 4 compares MSSM-Tower with SUSYHD and other public programs (Softsusy [8], Spheno [9], FeynHiggs (see [11] and references therein). In these proceedings we focus on two issues: the differences between the two fixed-order calculations in the $\overline{\mathrm{DR}}$-scheme, Softsusy and Spheno, and the differences between MSSM-Tower/SUSYHD and the fixed-order calculations.

- There are surprisingly large deviations between Softsusy and Spheno at high $M_{\mathrm{SUSY}}$, although both calculations are two-loop calculations in the same renormalization scheme. The reason is that both calculations differ in a subtlety, which causes different three- and higher loop terms being included in the calculation. This difference can be understood at the leading-logarithmic level and at leading order in the top Yukawa and strong gauge coupling. At this level, the fixed-order two-loop calculation implemented in Softsusy and 
Spheno yields

$$
\Delta M_{h}^{2}=-\frac{\beta_{\lambda}\left(m_{t}^{\overline{\mathrm{DR}}}\left(M_{\mathrm{SUSY}}\right)\right)^{4}\left(L-2 \alpha_{s}^{\mathrm{MSSM}}\left(M_{\mathrm{SUSY}}\right) \beta_{y} L^{2}\right)}{4 \pi v^{2}}
$$

where $\beta_{\lambda}, \beta_{y}$ are coefficients in the SM $\beta$ functions of $\lambda$ and the top Yukawa coupling, $m_{t}^{\overline{\mathrm{DR}}}\left(M_{\mathrm{SUSY}}\right), \alpha_{s}^{\mathrm{MSSM}}\left(M_{\mathrm{SUSY}}\right)$ are the running top mass and strong coupling in the MSSM and $L=\log \left(M_{\text {SUSY }} / M_{\text {weak }}\right)$. The running top mass is determined by the equation $m_{t}^{\overline{\mathrm{DR}}}\left(M_{\mathrm{SUSY}}\right)=$ $M_{t}+\Sigma_{t}^{\mathrm{MSSM}}$ in terms of the pole mass $M_{t}$. The difference is that Softsusy uses the pole mass $M_{t}$ as the momentum argument of the self energy $\Sigma_{t}^{\mathrm{MSSM}}$, while Spheno uses the $\overline{\mathrm{DR}}$ top mass. For $m_{t}^{\overline{\mathrm{DR}}}\left(M_{\mathrm{SUSY}}\right)$ itself, this difference amounts to a two-loop difference, and for $\Delta M_{h}^{2}$ it amounts to a three-loop difference. An analytic evaluation of the leading logarithms taken into account in EFT-calculations like FS-Tower or SUSYHD, in Softsusy, and in Spheno yields (in terms of $\alpha_{s}$, the strong gauge coupling in the SM at the scale $M_{t}$ )

$$
\Delta M_{h}^{2}=\frac{3 M_{t}^{4}}{v^{2} \pi^{2}}\left(L-16\left(\frac{\alpha_{s}}{4 \pi}\right) L^{2}+\left\{\frac{736}{9}_{\text {Softsusy }}, \frac{992}{9}{ }_{\text {Spheno }}, \frac{736}{3}_{\text {EFT }}\right\}\left(\frac{\alpha_{s}}{4 \pi}\right)^{2} L^{3}\right) .
$$

We find that both fixed-order calculations include different, but incorrect three-loop logarithms. Hence the difference between Softsusy and Spheno must be regarded as a measure of (a lower bound of) the theory uncertainty of both codes. The fact that in Fig. 4, which includes subleading and higher-order terms, Softsusy agrees better with the correct, EFT-result than Spheno, should be regarded as accidental.

- Now we focus on the behaviour of MSSM-Tower in Fig. 4. As is clear from the discussion of the previous figure, MSSM-Tower agrees well with SUSYHD at high $M_{\mathrm{SUSY}}$, but there are deviations at low $M_{\text {SUSY }}$. Now we also see that MSSM-Tower agrees well with all fixedorder calculations at low $M_{\text {SUSY }}$. This is true by construction, as a result of the choice of the pole-mass matching condition to determine $\lambda$ at the SUSY scale. Thus MSSM-Tower interpolates between the SUSYHD-result at high $M_{\text {SUSY }}$ and the fixed-order results at low $M_{\text {SUSY. }}$

In summary, the EFT-type calculation with pole-mass matching, implemented in MSSM-Tower in FlexibleSUSY is a promising and thoroughly tested approach towards precisely calculating the SM-like Higgs mass in SUSY models. By construction, the approach combines virtues of fixedorder and EFT calculations. The comparisons of the different calculations help in understanding the theory uncertainties of the calculations: the difference between MSSM-Tower and SUSYHD at low scales is mainly due to the missing power-suppressed terms in SUSYHD and thus should be interpreted as a theory uncertainty of SUSYHD; the difference between Softsusy and Spheno at high scales is due to different (but incorrect) logarithms taken into account accidentally due to the way the running top mass is evaluated — this difference should be regarded as a theory uncertainty of both codes.

As an outlook, the MSSM-Tower algorithm can and should be improved by carrying out the SUSY-scale matching at the two-loop instead of the one-loop level. It can further be applied to non-minimal SUSY models such as the MRSSM. 
As a final outlook on improvements of Higgs mass calculations in SUSY models we remark that all existing calculations rely on regularization by dimensional reduction being a SUSYpreserving regularization. This has been proven to be true in Ref. [12] up to the two-loop level in the gaugeless limit. In view of current efforts it would be crucial to study in detail whether the statement can be extended to higher orders or whether the regularization scheme should be modified.

\section{References}

[1] P. Diessner, J. Kalinowski, W. Kotlarski and D. Stöckinger, JHEP 1412 (2014) 124 [arXiv:1410.4791 [hep-ph]].

[2] P. Diessner, J. Kalinowski, W. Kotlarski and D. Stöckinger, Adv. High Energy Phys. 2015 (2015) 760729 doi:10.1155/2015/760729 [arXiv:1504.05386 [hep-ph]].

[3] P. Diessner, J. Kalinowski, W. Kotlarski and D. Stöckinger, JHEP 1603 (2016) 007 doi:10.1007/JHEP03(2016)007 [arXiv:1511.09334 [hep-ph]].

[4] P. Athron, J.-h. Park, T. Steudtner, D. Stöckinger, A. Voigt, to be published.

[5] G. D. Kribs, E. Poppitz and N. Weiner, Phys. Rev. D 78 (2008) 055010 [arXiv:0712.2039 [hep-ph]].

[6] P. Athron, J.-h. Park, D. Stöckinger and A. Voigt, Comput. Phys. Commun. 190 (2015) 139 [arXiv:1406.2319 [hep-ph]].

[7] F. Staub, Comput. Phys. Commun. 181, 1077 (2010) [arXiv:0909.2863 [hep-ph]]. Comput. Phys. Commun. 182, 808 (2011) [arXiv: 1002.0840 [hep-ph]]. Computer Physics Communications 184, pp. 1792 (2013) [Comput. Phys. Commun. 184, 1792 (2013)] [arXiv:1207.0906 [hep-ph]]. Comput. Phys. Commun. 185 (2014) 1773 [arXiv:1309.7223 [hep-ph]].

[8] B. C. Allanach, Comput. Phys. Commun. 143, 305 (2002) [hep-ph/0104145].

[9] W. Porod, Comput. Phys. Commun. 153 (2003) 275 [hep-ph/0301101]. W. Porod and F. Staub, Comput. Phys. Commun. 183 (2012) 2458 [arXiv:1104.1573 [hep-ph]].

[10] J. P. Vega and G. Villadoro, JHEP 1507 (2015) 159 doi:10.1007/JHEP07(2015)159 [arXiv:1504.05200 [hep-ph]].

[11] T. Hahn, S. Heinemeyer, W. Hollik, H. Rzehak and G. Weiglein, Phys. Rev. Lett. 112 (2014) no.14, 141801 doi:10.1103/PhysRevLett.112.141801 [arXiv:1312.4937 [hep-ph]]

[12] W. Hollik and D. Stöckinger, Phys. Lett. B 634 (2006) 63 doi:10.1016/j.physletb.2006.01.030 [hep-ph/0509298]. 